\title{
Supporting Information for \\ Functionalized Single-Walled Carbon Nanotubes and Nanographene Oxide to Overcome Antibiotic Resistance in Tetracycline-Resistant Escherichia coli
}

Jordan A. Carver, ${ }^{*}$ Audrey L. Simpson, ${ }^{*}$ Ria P. Rathi, ${ }^{*}$ Nerica Normil, ${ }^{*}$ Amy G. Lee, ${ }^{*}$ Madison D. Force, ${ }^{*}$ Katherine A. Fiocca, ${ }^{*}$ Christopher E. Maley, ${ }^{*}$ Kara M. DiJoseph, ${ }^{*}$ Abigail L.

Goldstein, ${ }^{\star}$ Amin A. Attari, ${ }^{\ddagger}$ Haley L. O’Malley, Jaclyn G. Zaccaro, Noël M. McCampbell, Christina A. Wentz, Jessica E. Long, Lilly M. McQueen, Francis J. Sirch, Broderick K. Johnson, Molly E. Divis, Matthew L. Chorney, Steven L. DiStefano, Holly M. Yost, Brandon L. Greyson, Emily A. Cid, Kyumin Lee, Codi J. Yhap, Michelle Dong, Dayna L. Thomas, Brittany E. Banks, Regan B. Newman, Jailene Rodriguez, Alix T. Segil, Justin A. Siberski, Anthony L. Lobo, ${ }^{a}$ and Mark D. Ellison ${ }^{b, *}$

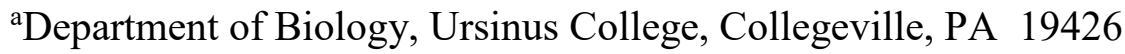

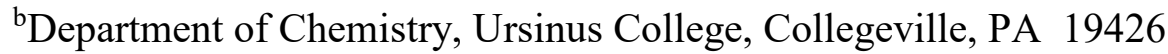

*Corresponding author. Department of Chemistry, Ursinus College, Collegeville, PA mellison@ursinus.edu

\section{Table of Contents}

Figure S1. TEM images of nanomaterials $\quad 2-4$

Figure S2. Length distributions of raw, cut, and PEGylated SWNTs 5

Figure S3. Size distributions of NGO and NGO-PEG 6-7

Figure S4. Relative growth of E. coli exposed to SWNT-PEG and 8-9 NGO-PEG

Table S1. Results of viable counts experiments 10-11

Figure S5. LDH assay results $12-13$

Figure S6. NMR spectra of nanomaterials $\quad$ 13-14

Table S2. Comparison of NMR peaks to control and literature values 14

Figure S7. FTIR spectra of nanomaterials $\quad 15$

Determination of Loading of TET on nanomaterials, including Figure 15-16 S8

Figure S9. Relative growth of $E$. coli after separate addition of $\quad 17$ nanomaterials and TET 


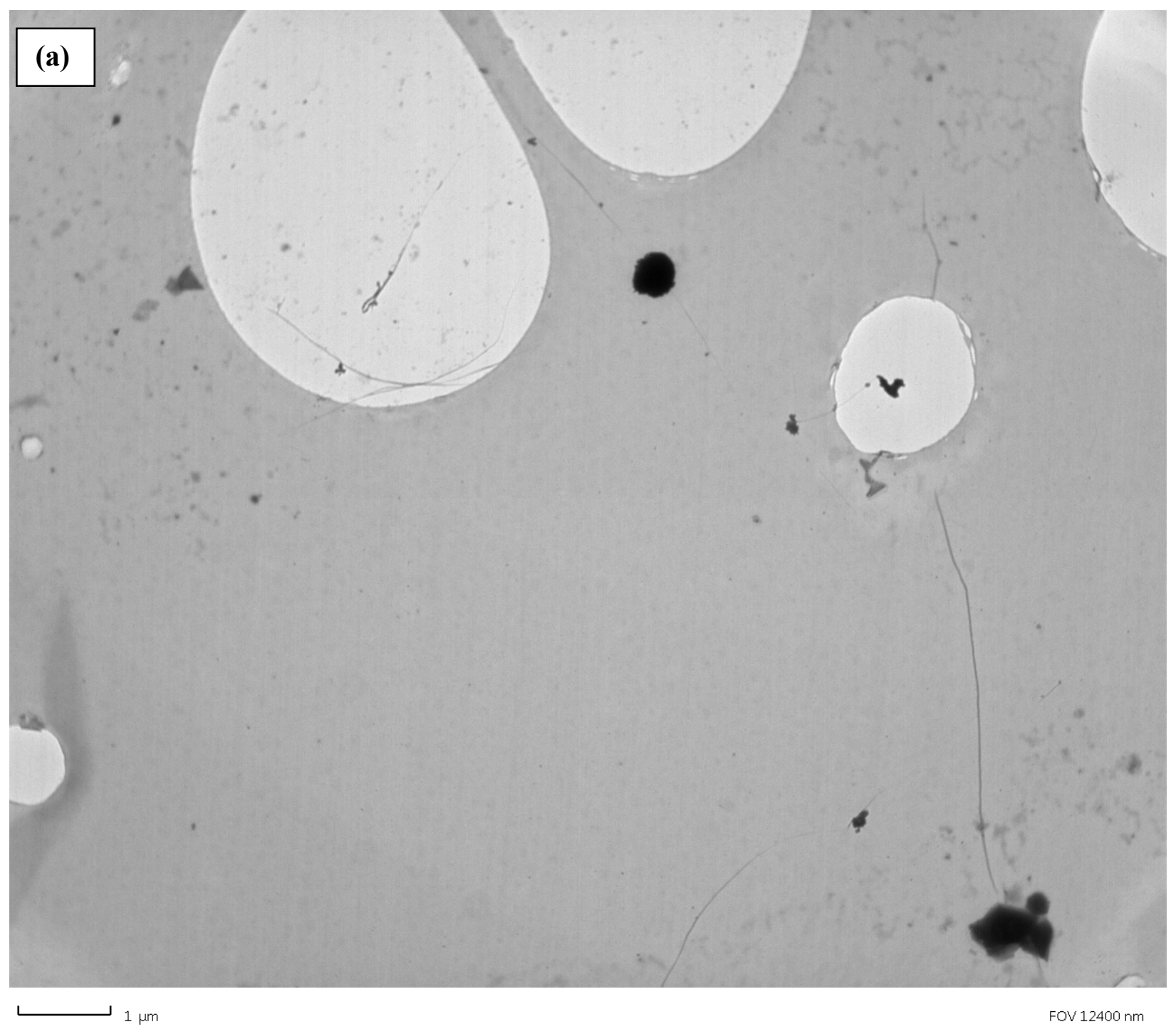




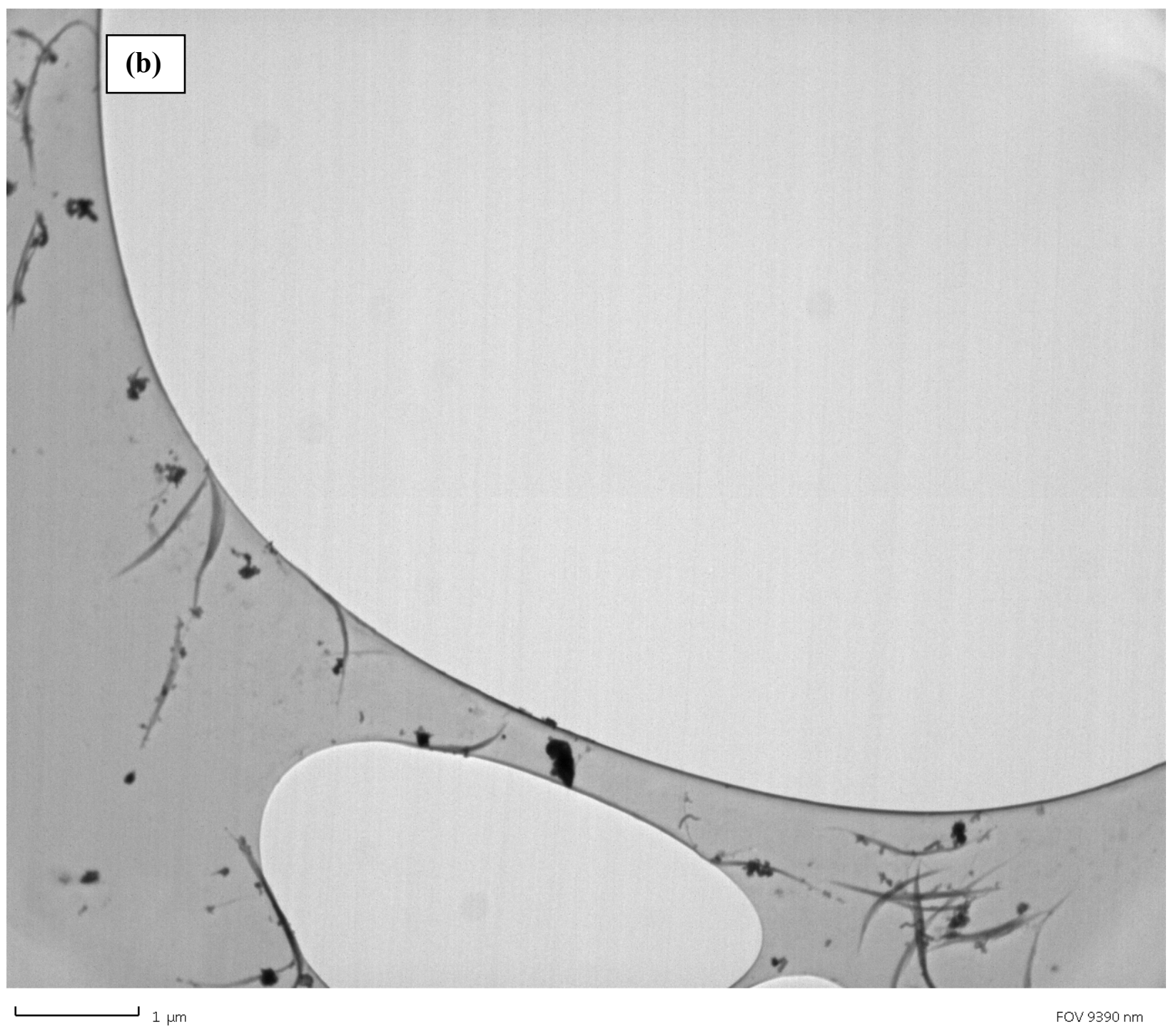




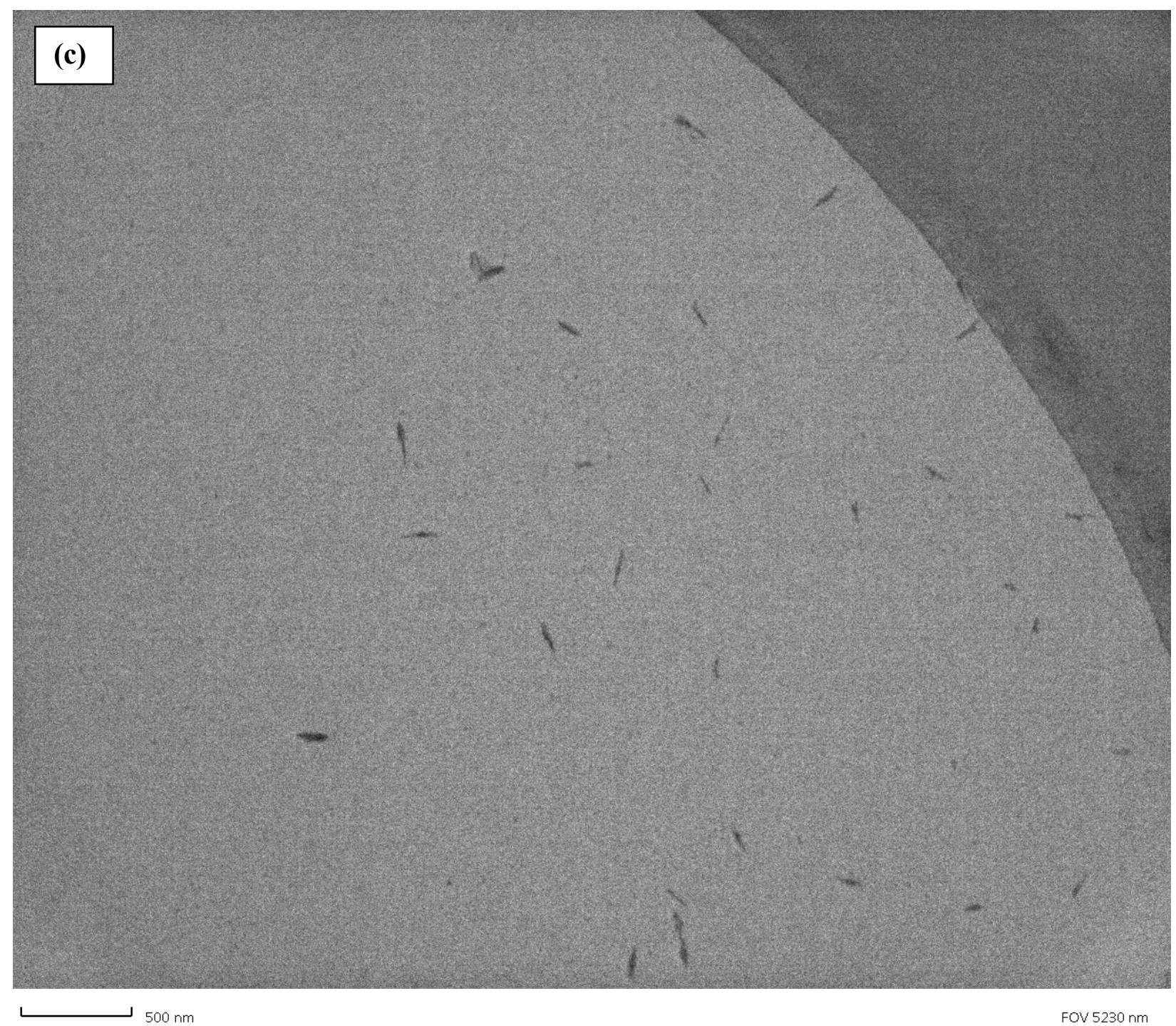

Figure S1. (a) Transmission electron microscope (TEM) image of as-purchased (raw) singlewalled carbon nanotubes (SWNTs). Scale bar is $1.0 \mu \mathrm{m}$. (b) TEM image of acid-cut SWNTs. Scale bar is $1.0 \mu \mathrm{m}$. (c) TEM image of nanographene oxide (NGO). Scale bar is $500 \mathrm{~nm}$. 

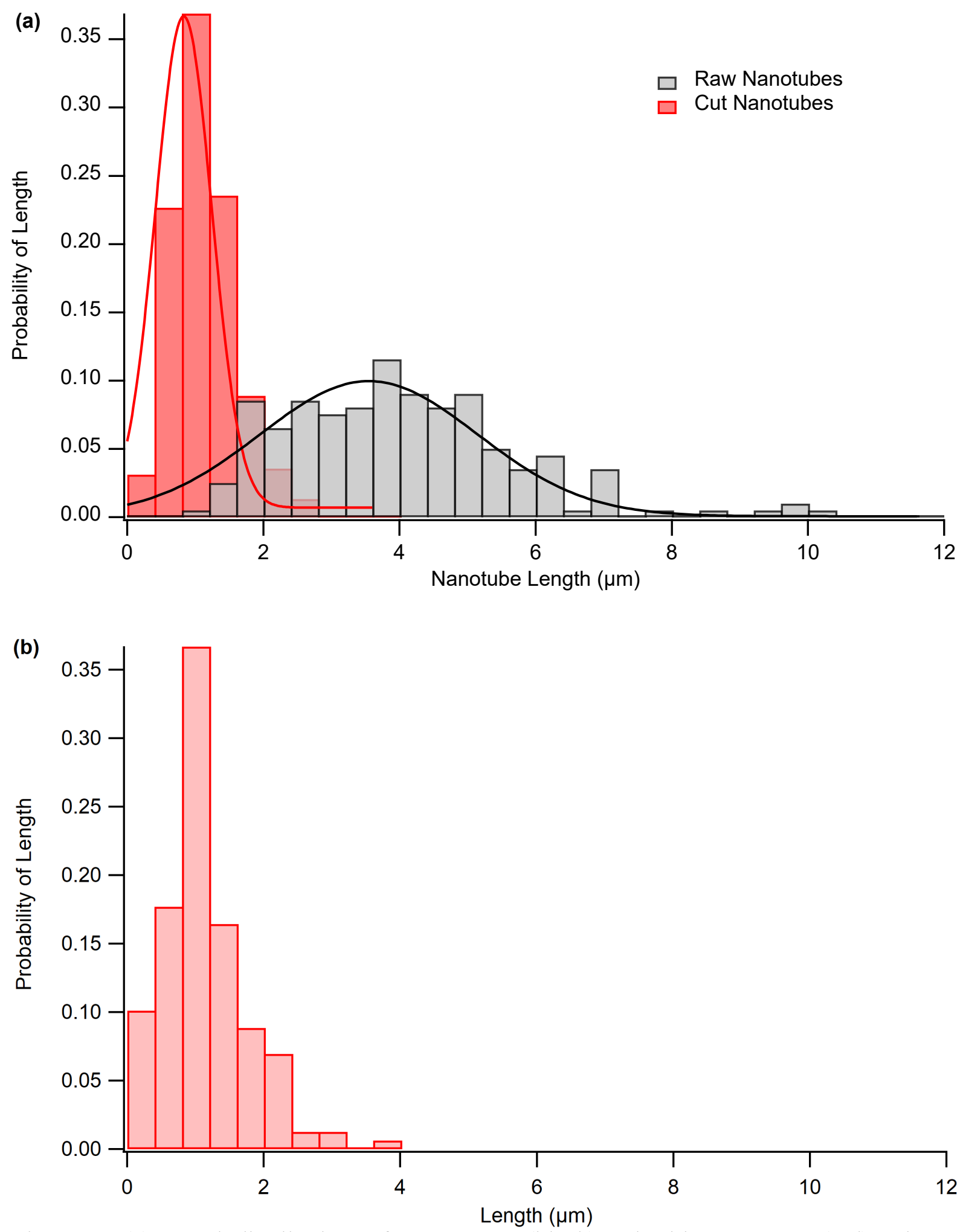

Figure S2. (a) Length distributions of raw SWNTs (black) and acid-cut SWNTs (red). The raw SWNTs are $4.0 \pm 1.7 \mathrm{um}$ long, whereas the cut SWNTs are $1.1 \pm 0.4 \mu \mathrm{m}$ long. The solid lines are Gaussian fits to guide the eye. (b) Length distribution of SWNT-PEG, which are $1.1 \pm 0.6$ $\mu \mathrm{m}$ long. 
(a)

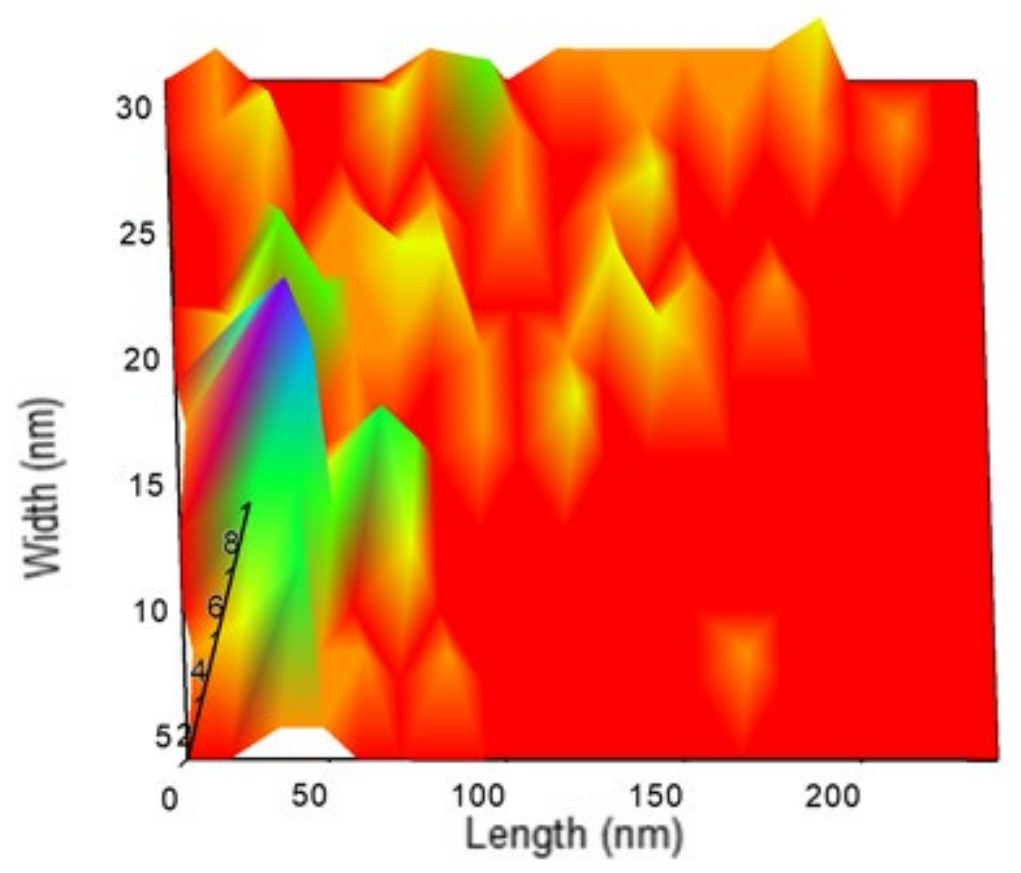


(b)

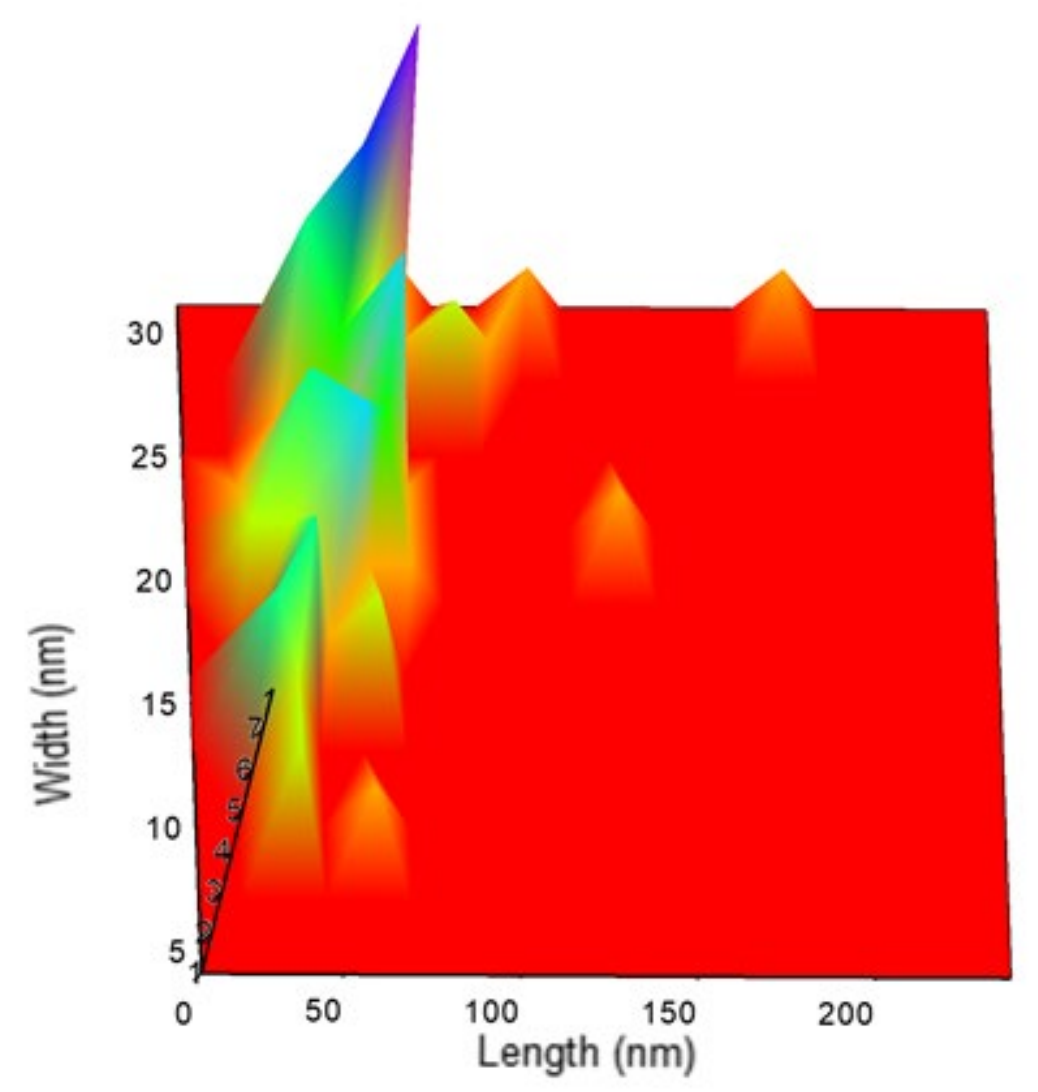

Figure S3. Size distributions of (a) NGO and (b) NGO-PEG. The colors indicate the probability of measuring that dimension. Red is zero probability, orange and yellow are low probability, green is medium probability, and blue is high probability. For NGO, the average dimensions are by $86 \pm 51 \mathrm{~nm}$ by $25 \pm 13 \mathrm{~nm}$, and for NGO-PEG, the average dimensions are $69 \pm 32 \mathrm{~nm}$ by 33 $\pm 16 \mathrm{~nm}$. 

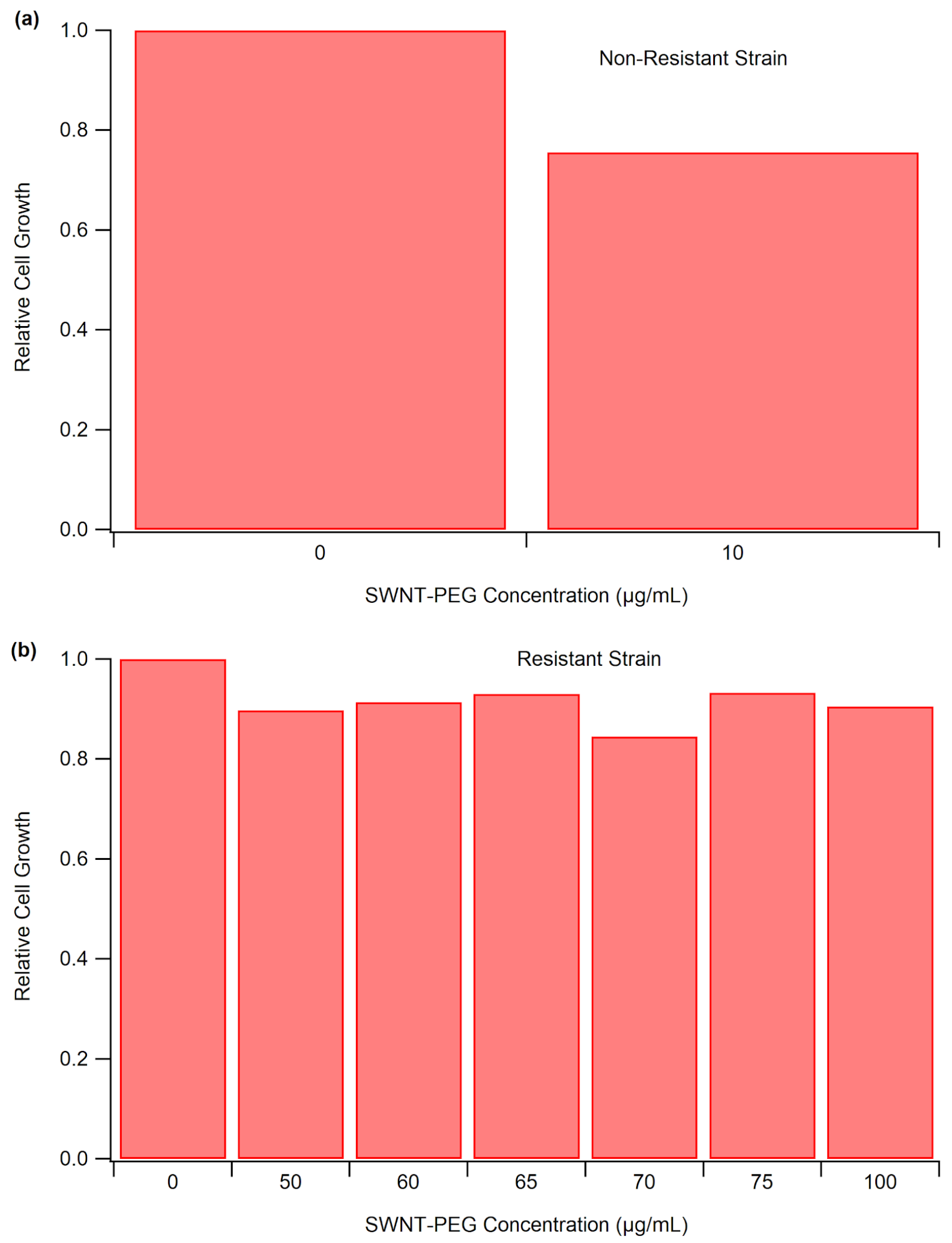

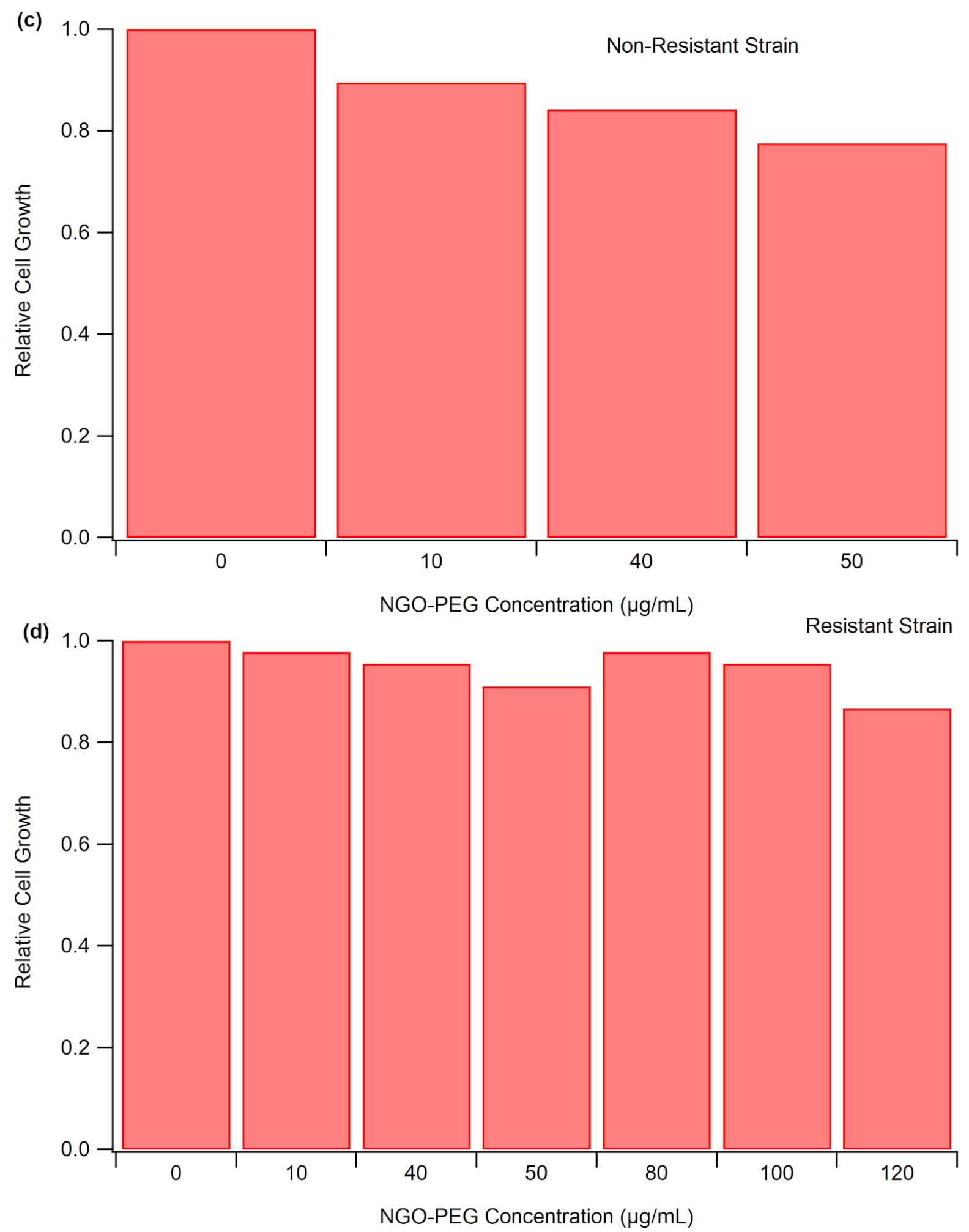

Figure S4. Relative bacteria growth of (a) DH5 $\alpha$ exposed to SWNT-PEG, (b) DH5 $\alpha-$ pBR322 exposed to SWNT-PEG, (c) DH5 $\alpha$ exposed to NGO-PEG, (d) DH5 $\alpha$-pBR322 exposed to NGOPEG. In each figure, the maximum concentration of nanomaterial is equal to or greater than its highest concentration in other experiments in this work. This demonstrates that the nanomaterials do not significantly inhibit bacterial growth. 
Table S1. Results of viable count experiments for DH5 $\alpha$ and DH5 $\alpha-$ pBR322 exposed to SWNT-PEG and NGO-PEG. (TNTC = too numerous to count) The number of counted colonies for bacteria exposed to nanomaterials is statistically the same as non-exposed bacteria, indicating that exposure to nanomaterials does not negatively impact the viability of the bacteria.

\begin{tabular}{|c|c|c|c|c|c|}
\hline DH5 $\alpha$ Exposure & \multicolumn{5}{|c|}{ Dilution Factor } \\
\hline \multirow{4}{*}{ Nothing } & 1 & $10^{-5}$ & $10^{-6}$ & $10^{-7}$ & $10^{-8}$ \\
\hline & TNTC & TNTC & TNTC & 42 & 4 \\
\hline & TNTC & TNTC & TNTC & 39 & 2 \\
\hline & TNTC & TNTC & TNTC & 28 & 4 \\
\hline \multirow{3}{*}{$\begin{array}{l}\text { SWNT-PEG } \\
(100 \mu \mathrm{g} / \mathrm{mL})\end{array}$} & TNTC & TNTC & TNTC & 41 & 6 \\
\hline & TNTC & TNTC & TNTC & 45 & 3 \\
\hline & TNTC & TNTC & TNTC & 49 & 7 \\
\hline \multirow{3}{*}{$\begin{array}{l}\text { NGO-PEG } \\
(100 \mu \mathrm{g} / \mathrm{mL})\end{array}$} & TNTC & TNTC & TNTC & 30 & 2 \\
\hline & TNTC & TNTC & TNTC & 42 & 10 \\
\hline & TNTC & TNTC & TNTC & 49 & 4 \\
\hline
\end{tabular}




\begin{tabular}{|c|c|c|c|c|c|}
\hline DH5 $\alpha-p B R 322$ & \multicolumn{5}{|c|}{ Dilution Factor } \\
\hline \multirow{4}{*}{ Nothing } & 1 & $10^{-5}$ & $10^{-6}$ & $10^{-7}$ & $10^{-8}$ \\
\hline & TNTC & TNTC & TNTC & 63 & 2 \\
\hline & TNTC & TNTC & TNTC & 53 & 6 \\
\hline & TNTC & TNTC & TNTC & 50 & 6 \\
\hline \multirow{3}{*}{$\begin{array}{l}\text { SWNT-PEG } \\
(100 \mu \mathrm{g} / \mathrm{mL})\end{array}$} & TNTC & TNTC & TNTC & 45 & 3 \\
\hline & TNTC & TNTC & TNTC & 44 & 2 \\
\hline & TNTC & TNTC & TNTC & 44 & 5 \\
\hline \multirow{3}{*}{$\begin{array}{c}\text { NGO-PEG } \\
(100 \mu \mathrm{g} / \mathrm{mL})\end{array}$} & TNTC & TNTC & TNTC & 51 & 8 \\
\hline & TNTC & TNTC & TNTC & 56 & 3 \\
\hline & TNTC & TNTC & TNTC & 63 & 1 \\
\hline
\end{tabular}



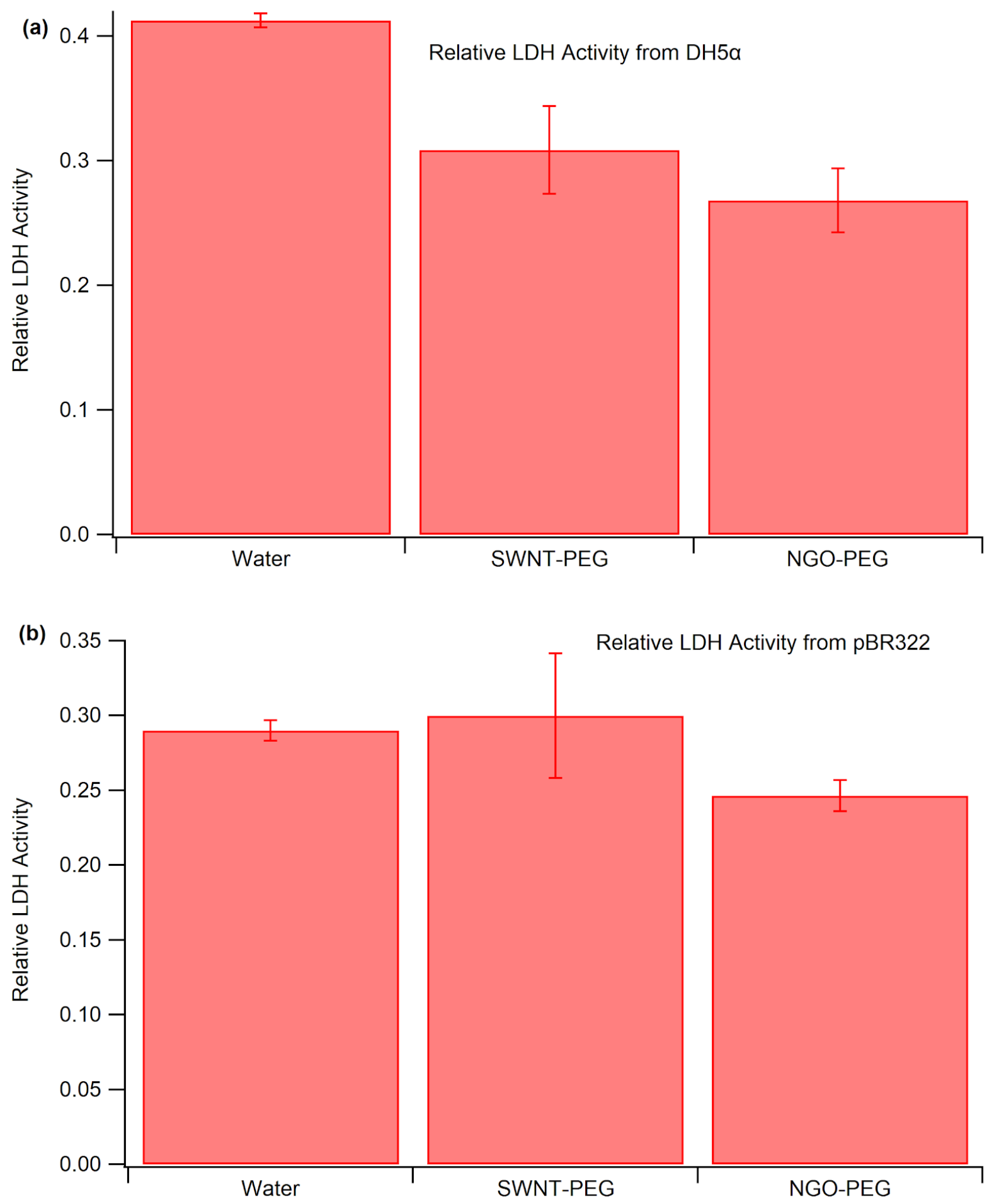

Figure S5. LDH activity assay results for (a) DH5 $\alpha$ and (b) DH5 $\alpha-$ pBR322 exposed to water control, SWNT-PEG, and NGO-PEG. In both cases, there is no evidence for increased LDH activity over the water control, indicating that the nanomaterials do not cause release of LDH from within the bacteria. 

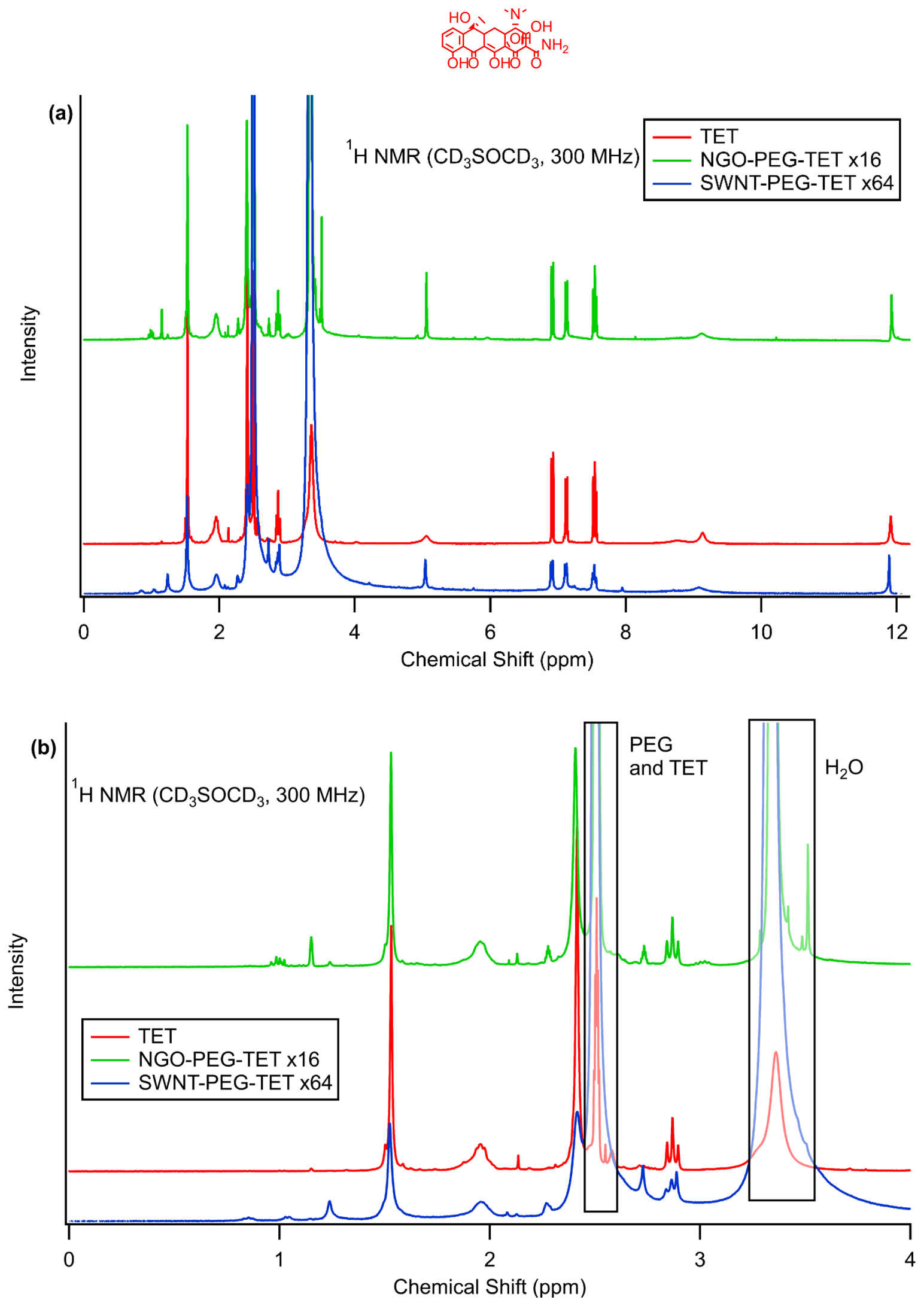


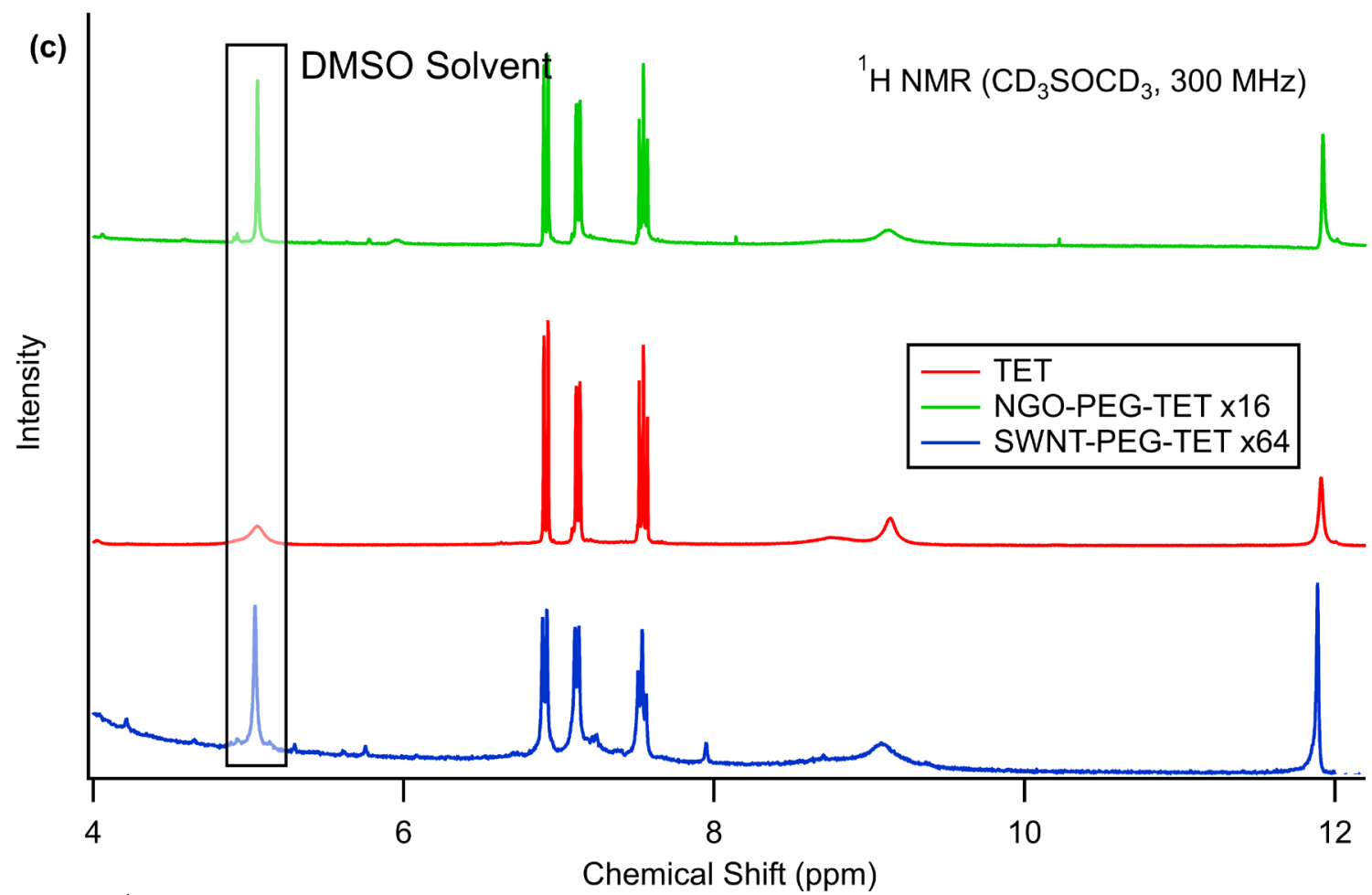

Figure S6. ${ }^{1} \mathrm{H}$ Nuclear Magnetic Resonance (NMR) spectrum of TET, SWNT-PEG, SWNTPEG-TET, and NGO-PEG-TET at $300 \mathrm{MHz}$ in $\mathrm{CD}_{3} \mathrm{COCD}_{3}$. (a) full spectrum, (b) low-shift region, (c) high-shift region. The peak at $5.04 \mathrm{ppm}$ is from the DMSO solvent. The peak at 3.33 ppm is from HOD that results from residual water in the sample after filtration and is also present in the DMSO solvent. This peak also coincides with a TET peak. The other peaks agree with literature values ${ }^{1}$ and the spectrum of our own TET. The peak at $2.51 \mathrm{ppm}$ is from the PEG. ${ }^{2}$ See Table S2 for more information.

Table S2. ${ }^{1} \mathrm{H}$ NMR chemical shifts for tetracycline from the literature, ${ }^{1}$ our own spectrum of TET, and TET attached to the nanomaterials. The peaks are characterized in the same manner as were those in the literature: $t=$ triplet, $d=$ doublet, $b s=$ broad singlet, $s=$ singlet. The excellent agreement between our peak values and those in the literature indicate that the TET is unchanged upon attachment to the nanomaterials.

\begin{tabular}{|l|c|c|c|l|l|l|l|l|l|}
\hline \multicolumn{1}{|c|}{ Compound } & \multicolumn{8}{c|}{ Chemical Shifts (ppm) } \\
\hline Literature $^{1}$ & $7.5 \mathrm{t}$ & 7.1 & $6.9 \mathrm{~d}$ & $1.5 \mathrm{bs}$ & $2.5 \mathrm{~s}$ & 9.1 & 8.6 & 11.9 & 3.3 \\
\hline TET & $7.55 \mathrm{t}$ & $7.13 \mathrm{~d}$ & $6.93 \mathrm{~d}$ & $1.53 \mathrm{~s}$ & $2.50 \mathrm{~s}$ & 9.14 & 8.70 & 11.91 & 3.36 \\
\hline SWNT-PEG-TET & $7.54 \mathrm{t}$ & $7.13 \mathrm{~d}$ & $6.92 \mathrm{~d}$ & $1.52 \mathrm{~s}$ & $2.50 \mathrm{~s}$ & 9.14 & 8.70 & 11.89 & 3.33 \\
\hline NGO-PEG-TET & $7.54 \mathrm{t}$ & $7.14 \mathrm{~d}$ & $6.93 \mathrm{~d}$ & $1.53 \mathrm{~s}$ & $2.51 \mathrm{~s}$ & 9.13 & 8.70 & 11.92 & 3.35 \\
\hline
\end{tabular}




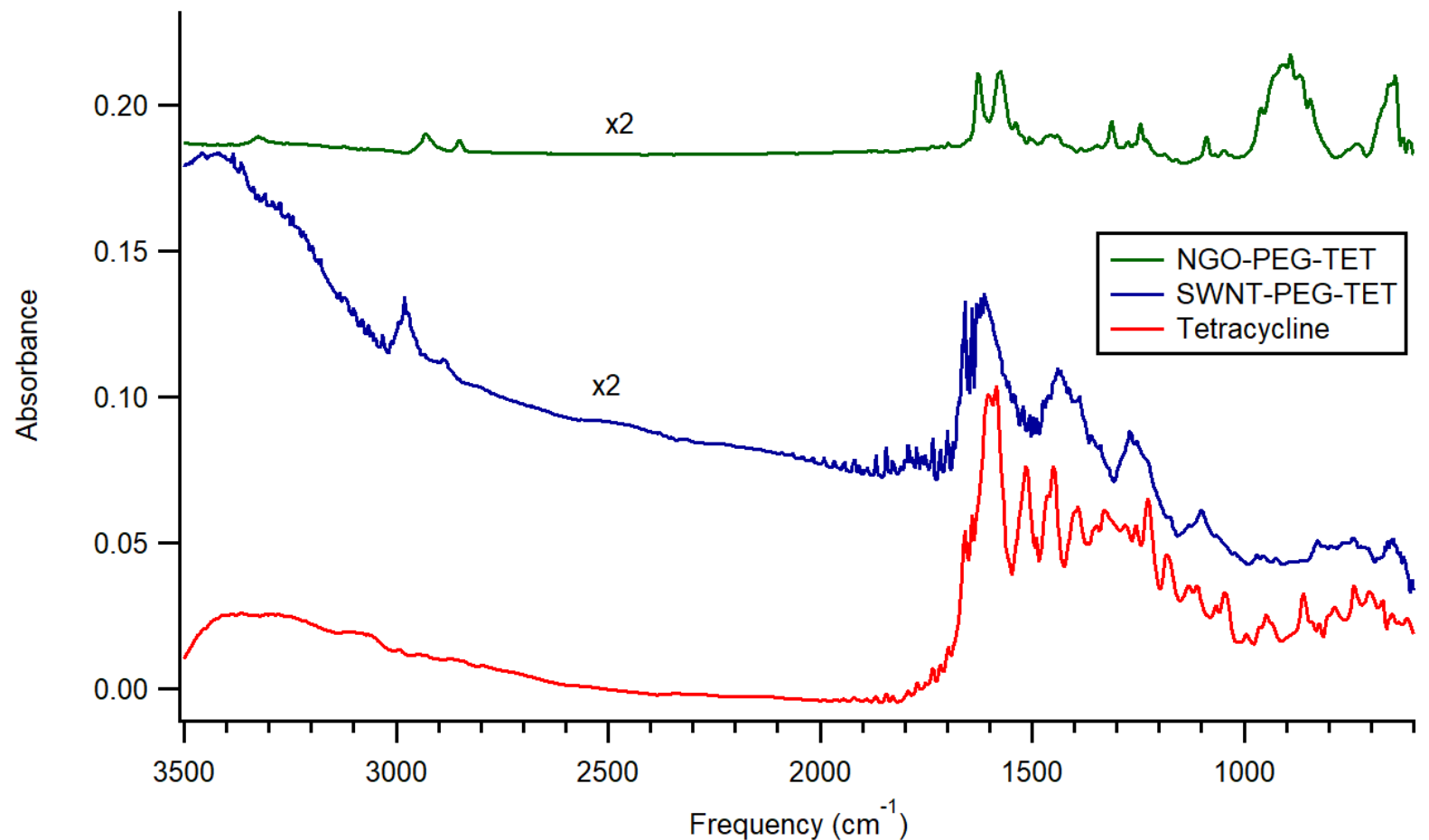

Figure S7. FTIR spectra of TET, SWNT-PEG-TET, and NGO-PEG-TET. The peak at $\sim 1650 \mathrm{~cm} \cdot$ indicates a $\mathrm{C}=\mathrm{O}$, which is present in TET, NGO and SWNT. The N-H stretch at $\sim 3300 \mathrm{~cm}^{-1}$, C-H peaks at $\sim 2900 \mathrm{~cm}^{-1}$, and the C-O peak at $\sim 1300 \mathrm{~cm}^{-1}$ all confirm the presence of PEG on the NGO and SWNT. The TET peaks are broadened on the SWNT and broadened and shifted on NGO as a result of adsorption. These results, together with the NMR results, indicate the presence of TET on the nanomaterials.

\section{Determination of TET Loading of SWNT-PEG and NGO-PEG}

As described in the Methods section, SWNT-PEG or NGO-PEG was stirred in a TET solution at $4{ }^{\circ} \mathrm{C}$ overnight. The nanomaterials were filtered out, and the filtrate was analyzed by UV-Vis spectroscopy to determine the amount of TET in the filtrate. This amount was subtracted from the original amount of TET in the reaction mixture to yield the amount of TET attached to the nanomaterial. Data and calculations for SWNT-PEG are shown below, and the same process was performed for NGO-PEG. For this experiment, $0.0741 \mathrm{~g}$ of SWNT-PEG was mixed with $0.1112 \mathrm{~g}$ of TET and then filtered.

Standard tetracycline solutions of known concentrations were made through serial dilutions. The absorbances of these solutions at $358 \mathrm{~nm}$ were measured and graphed. The data fit very well to a line, and the equation of the line was used to calculate the concentration of TET in the filtrate, as described below. 


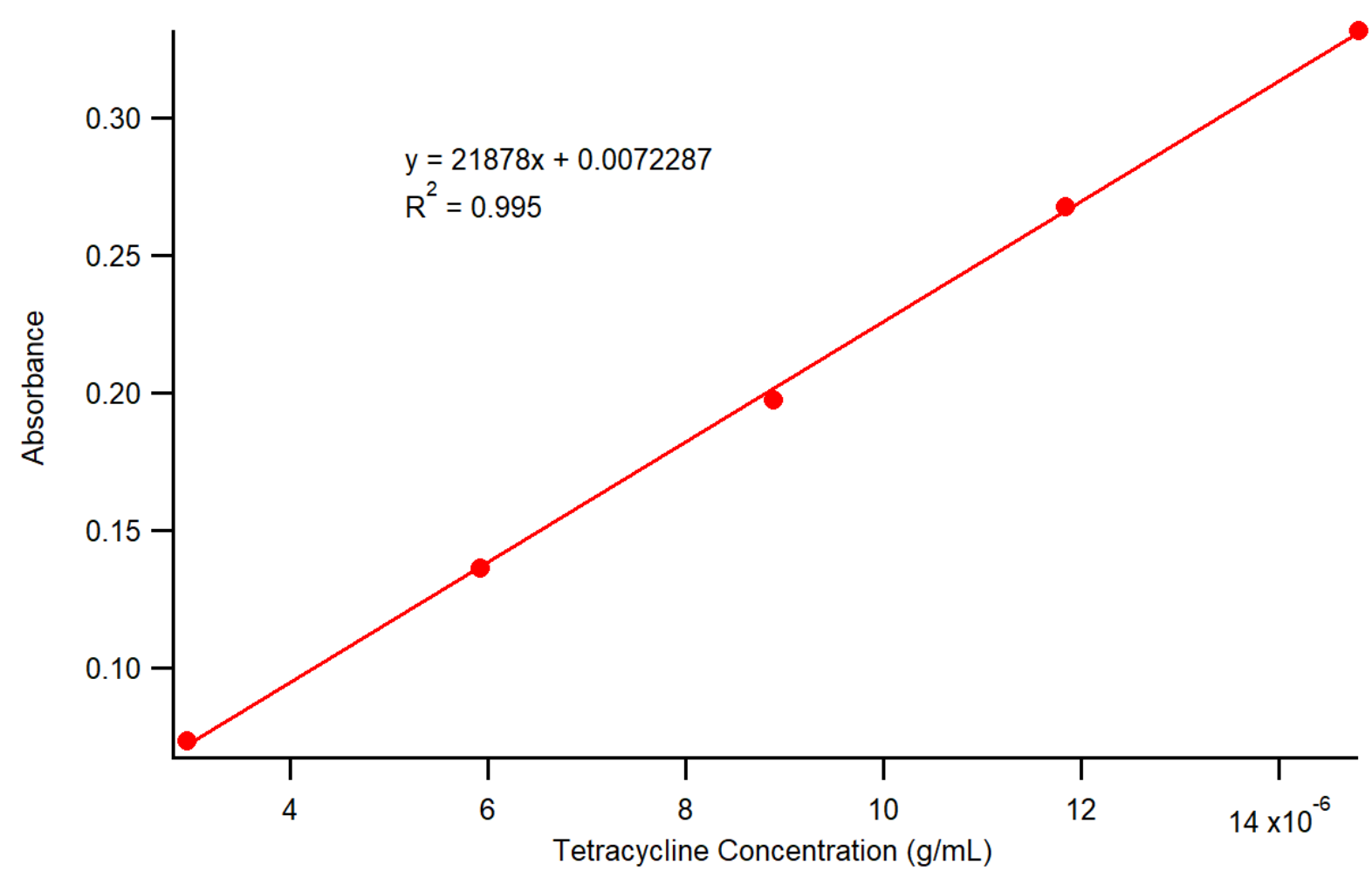

Figure S8. Calibration curve of standard tetracycline solutions.

The filtrate had a volume of $3.9 \mathrm{~mL} .1 .00 \mathrm{~mL}$ of it was pipetted into a $50.0-\mathrm{mL}$ volumetric flask and diluted to $50.0 \mathrm{~mL}$. The absorbance of this solution at $358 \mathrm{~nm}$ was measured to be 0.51636 .

$$
\begin{gathered}
0.51636=21878 \mathrm{x}+0.0072287 \\
\mathrm{x}=2.3271 \times 10^{-5} \mathrm{~g} / \mathrm{mL} \text { This is the concentration of the diluted TET. }
\end{gathered}
$$

Multiply by $50: \mathrm{x}=0.0011636 \mathrm{~g} / \mathrm{mL}$ This is the concentration of TET in the filtrate.

Multiply by $3.9 \mathrm{~mL}$, the volume of the filtrate: $\mathrm{x}=0.0045 \mathrm{~g}$ This is the mass of TET in the filtrate.

TET attached $=0.1112 \mathrm{~g}-0.0045 \mathrm{~g}=0.107 \mathrm{~g}$

Mass Percent of TET in SWNT-PEG-TET $=0.107 \mathrm{~g}$ TET0.107 g TET $+0.0741 \mathrm{~g}$ SWNTPEG $100 \%=59.1 \%$ TET

For five trials, the average mass percent of TET in SWNT-PEG-TET was 55\%. For NGO-PEGTET, the average was $50 \%$. 

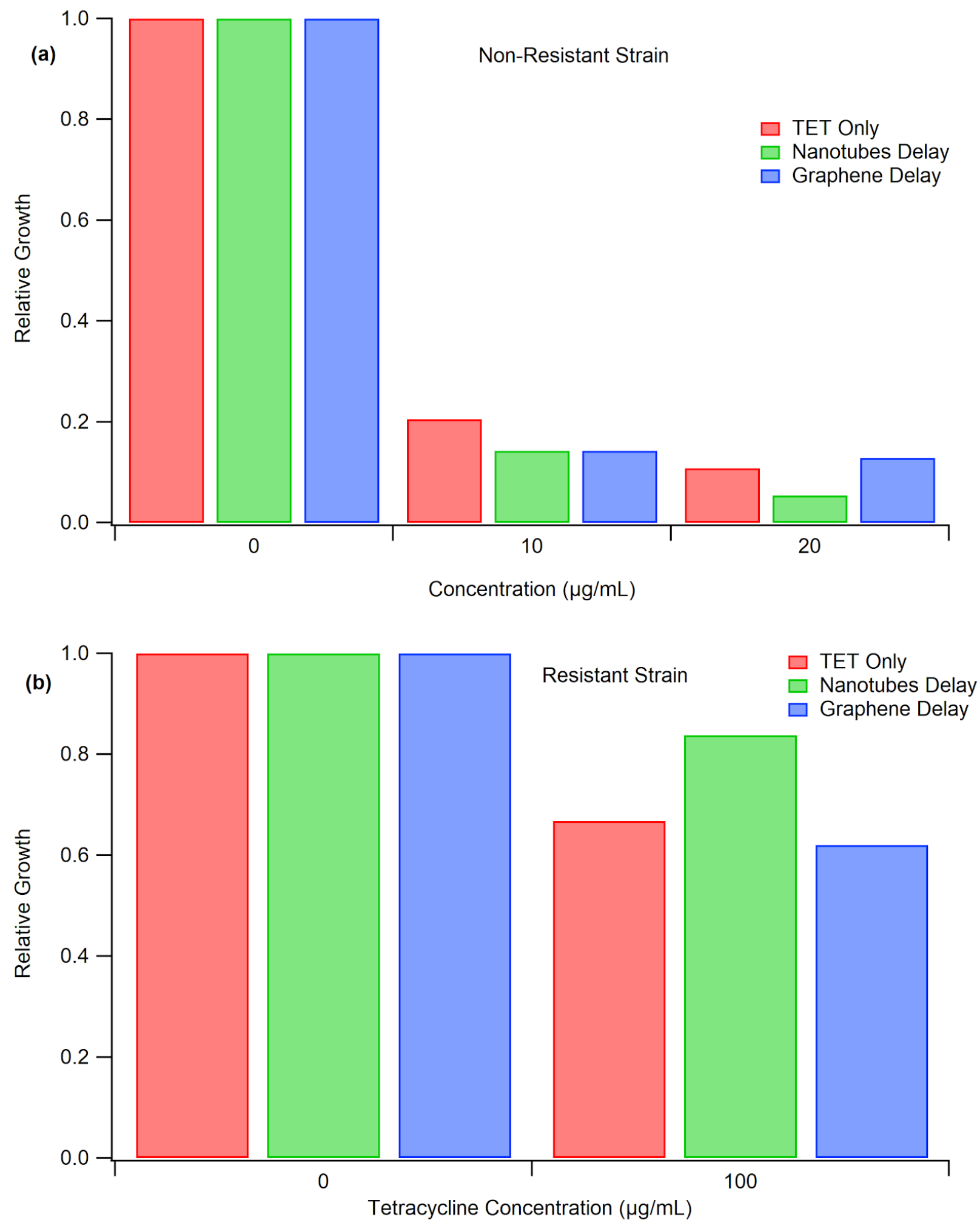

Figure S9. Relative survival after 24-h incubation for separate additions of SWNT-PEG and NGO-PEG and then TET 1 h later for (a) DH5 $\alpha$ and (b) DH5 $\alpha$-pBR322 strains of bacteria. The TET inhibited the DH5 $\alpha$ strain, indicating that the presence of the nanomaterials does not hinder the action of the TET. However, the DH5 $\alpha$-pBR322 strain was only slightly inhibited. The 
extent of inhibition of the resistant strain is similar to that of just TET, indicating that adding nanomaterials separately from the TET does not lead to significant inhibition of the resistant bacteria.

\section{References}

1. Casy, A. F.; Yasin, A. The identification and stereochemical study of tetracycline antibiotics by ${ }^{1} \mathrm{H}$ nuclear magnetic resonance spectroscopy. Journal of Pharmaceutical and Biomedical Analysis. 1, 28192 (1983).

2. Dust, J. M.; Fang, Z. H.; Harris, J. M. Proton NMR characterization of poly(ethylene glycols) and derivatives. Macromolecules. 23, 3742-6 (1990). 10.1021/ma00218a005 